\title{
Inhibition of syndecan-4 reduces cartilage degradation in murine models of osteoarthritis through the downregulation of HIF-2a by miR-96-5p
}

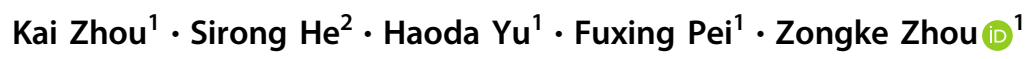

Received: 5 October 2020 / Revised: 27 March 2021 / Accepted: 29 March 2021 / Published online: 13 April 2021

(c) The Author(s), under exclusive licence to United States and Canadian Academy of Pathology 2021

\begin{abstract}
The membranous receptor syndecan-4 (SDC-4) and the nuclear transcription factor hypoxia-induced factor-2 $\alpha$ (HIF-2 $\alpha$ ) play critical roles in the pathogenesis of osteoarthritis (OA). The aim of this study was to determine whether SDC-4 inhibition downregulates HIF-2a expression by microRNA-96-5p (miR-96-5p) in murine chondrocyte and cartilage tissue. The OA model was induced surgically in mice, and SDC-4 polyclonal antibody, HIF-2 $\alpha$ small interfering RNA (siRNA) and its control, miR-96-5p mimics and its scrambled controls or anti-miR-96-5p and its control were then injected into the knee joints. At 2 and 4 weeks after surgery, OA progression was evaluated microscopically, histologically, radiographically and immunohistochemically in these mice. Real-time polymerase chain reaction (RT-PCR) and western blotting were performed after treating with antibody and transfecting with miRNA mimic or siRNA to determine their effects on OA-related mediators. The potential miRNAs related to OA development were identified by using miRNA microarray analysis. Whether miRNAs play a pivotal role in OA development in vivo or in vitro was also investigated. MiR-96-5p expression was upregulated by SDC-4-specific antibodies in chondrocytes and cartilage tissue, and miR-96-5p directly targeted the 3'-UTR of HIF- $2 \alpha$ to inhibit HIF- $2 \alpha$ signaling in murine chondrocytes. Moreover, we demonstrated that anti-SDC-4-attenuated IL$1 \beta$-induced chondrocyte hypertrophy and cartilage degradation by inhibiting HIF-2 $\alpha$ signaling by a miR-96-5p-dependent mechanism. Our study revealed that the inhibition of SDC-4 exerts its effects on both cartilage homeostasis and the chondrocyte hypertrophy phenotype by inducing miR-96-5p expression, which results in targeting HIF-2 $\alpha 3^{\prime}-$ UTR sequences and inhibiting HIF- $2 \alpha$ in murine cartilage tissue and chondrocytes.
\end{abstract}

\section{Introduction}

Osteoarthritis (OA) is a chronic joint disease characterized by progressive cartilage destruction, synovial hyperplasia and osteophyte formation [1,2]. Chondrocytes participate in cartilage destruction through proinflammatory mediator synthesis, such as interleukin-1 (IL-1), matrix metalloproteinases

Supplementary information The online version contains supplementary material available at https://doi.org/10.1038/s41374021-00595-5.

Zongke Zhou

Zhouzongke2016@163.com

1 Department of Orthopedics, West China Hospital, Sichuan University, Chengdu, PR China

2 Department of Immunology, Chongqing Medical University, Chongqing, PR China
(MMPs) and A disintegrin and metalloproteinase with thrombospondin motifs (ADAMTS) [3-5]. The process of cartilage repair has been demonstrated to be a type of noxious stimulation that induces a chondrocyte phenotypic change upon an imbalance in the metabolism of decomposition and synthesis $[6,7]$.

Syndecans, a family of cell-surface heparan sulfate proteoglycans, act as membranous receptors that interact with a variety of extracellular matrix molecules [8, 9]. Syndecan-4 (SDC-4) expression has been shown to be significantly increased in OA chondrocytes and is implicated in cartilage matrix degradation [5, 10-12]. Some studies have demonstrated that intraarticular injections of SDC-4 antibodies can decrease cartilage degradation by downregulating the expression of MMP-3 and the activity of ADAMTS5 in hypertrophic chondrocytes [10, 13]. SDC-4 deficiency has been shown to have a protective role in OA, which is characterized by decreased MMP-13 activity [14, 15]. However, the mechanisms that regulate the expression of 
SDC-4 and its pathophysiological function are unknown. In recent years, it has been shown that hypoxia can influence SDC-4 mRNA and protein expression in nucleus pulposus cells from healthy intervertebral discs [16].

Similar to the intervertebral disc, articular cartilage is maintained in a low oxygen environment throughout life; therefore, chondrocytes adapt to these hypoxic conditions [17]. Hypoxia-induced factors, a family of nuclear transcription factors, play a central role in mediating the histocyte response to hypoxic microenvironments [18-20]. Hypoxia-induced factor- $2 \alpha$ (HIF- $2 \alpha)$ is mainly expressed in highly differentiating chondrocytes as a key catabolic transcription factor that mediates the hypertrophic differentiation of chondrocytes and the cartilage degradation of osteoarthritic cartilage in humans and mice [4, 21-23]. The overexpression of HIF-2 $\alpha$ causes progressive cartilage damage and the upregulation of multiple degradative enzymes, including MMP-13 [22]. Although both SDC-4 and HIF-2 $\alpha$ participate in OA pathogenesis by influencing chondrocyte hypertrophic differentiation and cartilage degradation, the relationship between the membranous receptor SDC-4 and the nuclear transcription factor HIF- $2 \alpha$ remains unclear.

Importantly, miRNAs have recently emerged as key regulators of OA development and as mediators of chondrocyte hypertrophic differentiation and cartilage degradation in humans and mice [24-29]. In this study, we investigated whether the inhibition of SDC-4 in murine cartilage tissue and chondrocytes could alter the expression profile of miRNAs, and we found that miR-96-5p expression was upregulated after inhibiting SDC-4 expression. Moreover, the results demonstrate that the inhibition of SDC-4 exerts its effects on both cartilage homeostasis and the chondrocyte hypertrophy phenotype by inducing miR-96-5p expression, which results in targeting HIF-2 $\alpha$ 3'-UTR sequences and inhibiting HIF- $2 \alpha$ signaling in murine cartilage tissue and chondrocytes. Therefore, this approach provides a potential new strategy to prevent the progression of osteoarthritis.

\section{Materials and methods}

\section{Mouse chondrocyte stimulation in vitro}

Primary chondrocytes were isolated from the knee joint cartilage of 5-6-day-old mice as previously described in detail [30]. Cartilage samples were washed in calcium-free and magnesium-free PBS and finely ground. Chondrocytes were obtained by digesting the articular cartilage with $0.2 \%$ pronase (Sigma, USA) for $1 \mathrm{~h}$, followed by digestion with $0.2 \%$ collagenase (Sigma, USA) for $3 \mathrm{~h}$ at $37^{\circ} \mathrm{C}$ in highglucose Dulbecco's-modified Eagle's medium (DMEM, Life Technologies, USA) containing an antibiotic solution
(100 U/ml penicillin and $100 \mathrm{mg} / \mathrm{ml}$ streptomycin; Life Technologies). Undigested cartilage was removed with a $70 \mu \mathrm{m}$ nylon mesh (Cell Strainer, Falcon), and the chondrocytes were collected by centrifugation, washed twice, and resuspended in DMEM with $10 \%$ fetal bovine serum (Life Technologies, USA).

Chondrocytes were plated in six-well microculture plates (at a concentration of $10^{5}$ cells/well) and transfected with the following constructs and reagents [1]: miR-96-5p mimics or miRNA scrambled controls miR-96-5p (control mimics) at a final concentration of $30 \mathrm{nM}$ using oligofectamine (Invitrogen, USA) [2]; the antisense oligonucleotide inhibitor of miR-96-5p (anti-miR-96-5p) or the scrambled oligonucleotide (anti-miRNA control) at a final concentration of $50 \mathrm{nM}$ using DharmaFECT3 transfection reagent (Dharmacon, USA); and [3] HIF-2 $\alpha$ small interfering RNA (siRNA) or a control siRNA (Santa Cruz Biotechnology, USA) at a final concentration of $100 \mathrm{nM}$ using RNAi transfection reagent. Twenty-four hours after transfection, the cells were pretreated with a polyclonal SDC-4-specific antibody (100 mM, Becton Dickinson, USA) for $2 \mathrm{~h}$ and then stimulated either with or without recombinant human IL-1 $\beta$ ( $10 \mathrm{ng} / \mathrm{ml}, \mathrm{R} \& D$ Systems, USA) for $4 \mathrm{~h}$. Real-time PCR was used to detect the expression levels of miR-140-5p, miR-96-5p, miR-496, miR-26a, miR335, miR-16, miR-152-3p, miR-125b, miR-20a, miR-149, HIF-2 $\alpha$, CHMP2B, BASP1, CTNND1 EPHA3, MPP1, MSN, PLOD2, RPS6KA6, RUNDC3B, ST7, XKR4, COLX, SOX9, MMP-13, Ihh, RUNX2, and ADAMTS5. Western blotting was used to detect the expression of HIF- $2 \alpha$.

\section{In vivo experiments}

This study was approved by the Institutional Animal Care and Use Committee of Sichuan University and all experiments were performed in accordance with the National Institutes of Health guidelines for the care and use of laboratory animals (NIH Publications No. 8023, revised 1978). In all, 8-10-week-old male ICR mice (weighing 25-35 g) were purchased from Central Lab. Animal, Inc., of Sichuan University and maintained in a specific pathogenfree environment. For the induction of osteoarthritis in mice, we performed microsurgery on the left leg of anesthetized mice as described in a previous report, transected the anterior cruciate ligament, and removed the anterior corner of the medial meniscus [31]. $10 \mu \mathrm{l}$ of anti-SDC-4 polyclonal antibody (Becton Dickinson, USA) or saline was injected into the mouse knee joint cavity twice a week. In the following experiments, the mice were administered $100 \mathrm{nM}$ HIF- $2 \alpha$ siRNA or a control siRNA (Santa Cruz), $50 \mathrm{nM}$ miR-96-5p mimics or miRNA scrambled controls, or $50 \mathrm{nM}$ anti-miR-96-5p or an anti-miRNA control in the knee joint cavity twice a week. We measured the general 

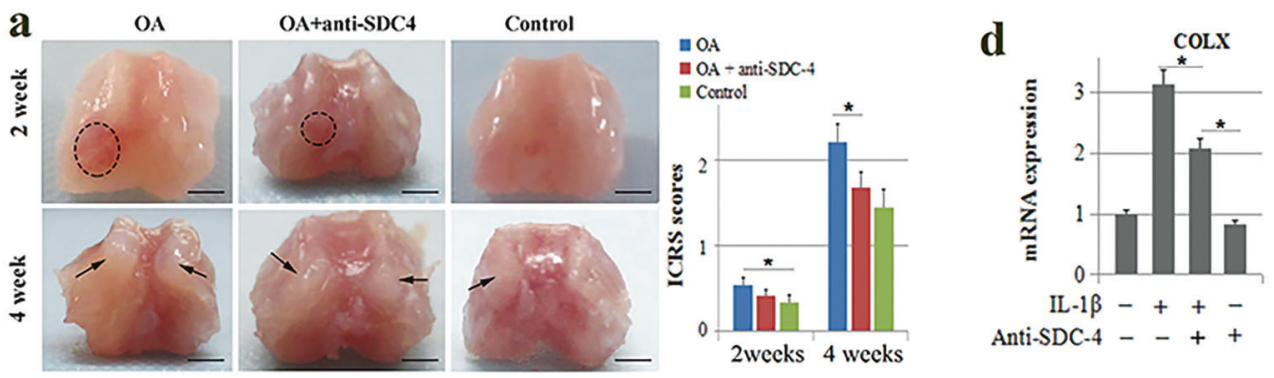

f
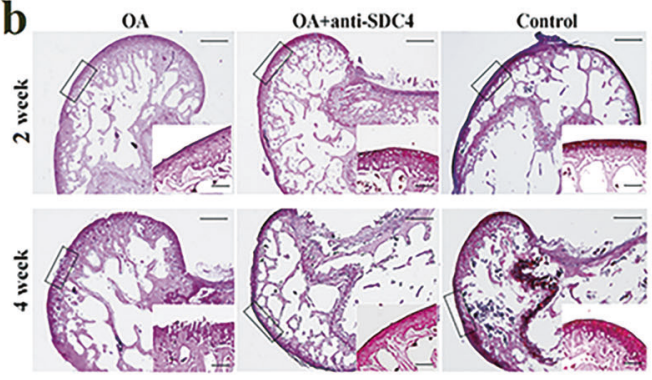

a $\mathrm{OA}+\operatorname{anti-SDC-4}$

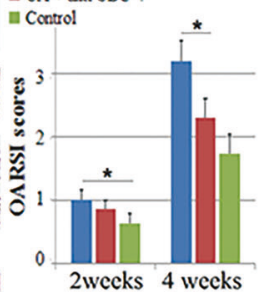

C
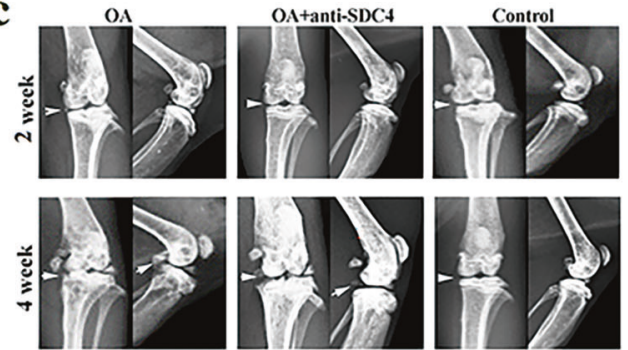

$D A$

aA + anti-SDC-4

a Control

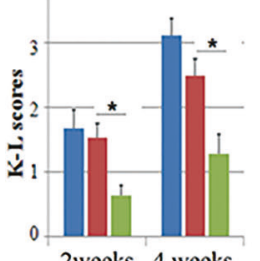

h

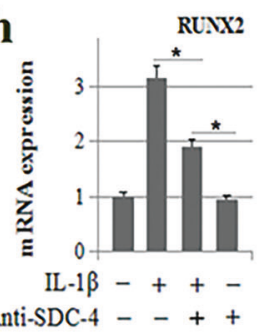

e

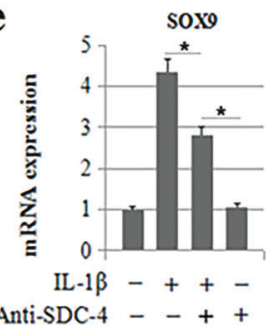

g

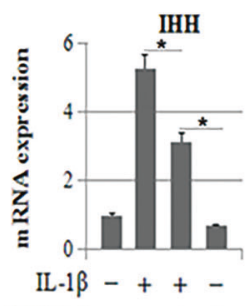

Anti-SDC-4 - + +

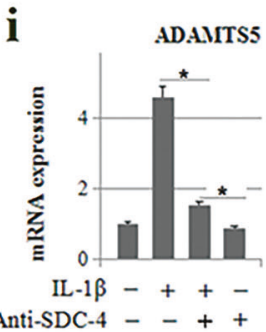

Fig. 1 The effect of SDC-4-specific antibodies on murine osteoarthritis and chondrocyte hypertrophy. Overall, 8-10-week-old male ICR mice (weighing 25-35 g) were transected into the meniscotibial ligament, the medial parts of the meniscus were removed, and syndecan-4-specific antibody or an equivalent volume of normal saline was injected into the knee joint cavity. Morphological (a), histological (b), and radiographic (c) analyses of the femoral condyles were performed using a digital camera, safranin $\mathrm{O}$ staining, and $\mathrm{X}$-ray, respectively, at 2 and 4 weeks post operation. a The cartilage lesions were graded on a scale from the International Cartilage Repair Society (ICRS). The dotted circles indicate wear area. The black arrows indicate chondrofibrosis. Bar $=2 \mathrm{~mm}$. b The joint lesions were graded

pathologic, radiographic, histological, and immunohistochemical characteristics at 2 and 4 weeks post operation. MicroRNAs were identified by miRNA microarray analysis (Agilent Technologies, USA) in cartilage tissue from OA mice treated with SDC-4-specific antibodies relative to control OA mice.

\section{Statistical analysis}

All quantitative data are presented as the mean \pm standard deviation (SD). The independent $t$-test and Mann-Whitney $U$ test were used to identify the significant differences between two groups as appropriate. For multiple group comparisons, one-way analysis of variance was performed using the (Osteoarthritis Research Society International) OARSI scoring system. Big picture bar $=500 \mu \mathrm{m}$; small picture bar $=100 \mu \mathrm{m}$. c The radiographic changes were graded using the Kellgren-Lawrence $(\mathrm{K}-\mathrm{L})$ scales. The white arrows indicate the degree of joint space narrowing and osteophytes. Primary mouse chondrocytes were pretreated with the syndecan-4-specific antibody $(100 \mathrm{mM})$ for $2 \mathrm{~h}$ and then stimulated with or without recombinant human IL-1 $\beta(10 \mathrm{ng} / \mathrm{ml})$ for $4 \mathrm{~h}$. Expression of transcription factor and hypertrophy phenotypic markers, including COL-X (d), SOX9 (e), MMP-13 (f), IHH (g), RUNX2 (h), and ADAMTS5 (i), was determined by RT-PCR analyses in chondrocytes. The values are expressed as the mean \pm SD from three independent experiments. $* P<0.05$.

with Tukey's post hoc analysis when the data exhibited equal variance and Dunnett's post hoc test when the data exhibited unequal variance. $P$ values $<0.05$ were considered statistically significant.

\section{Results}

\section{Anti-SDC-4 decreases cartilage degradation and inhibits IL-1 $\beta$-induced chondrocyte hypertrophy}

First, we investigated whether anti-SDC-4 could attenuate cartilage degradation in osteoarthritic mice. The general morphology of the femoral condyles showed that anti-SDC- 


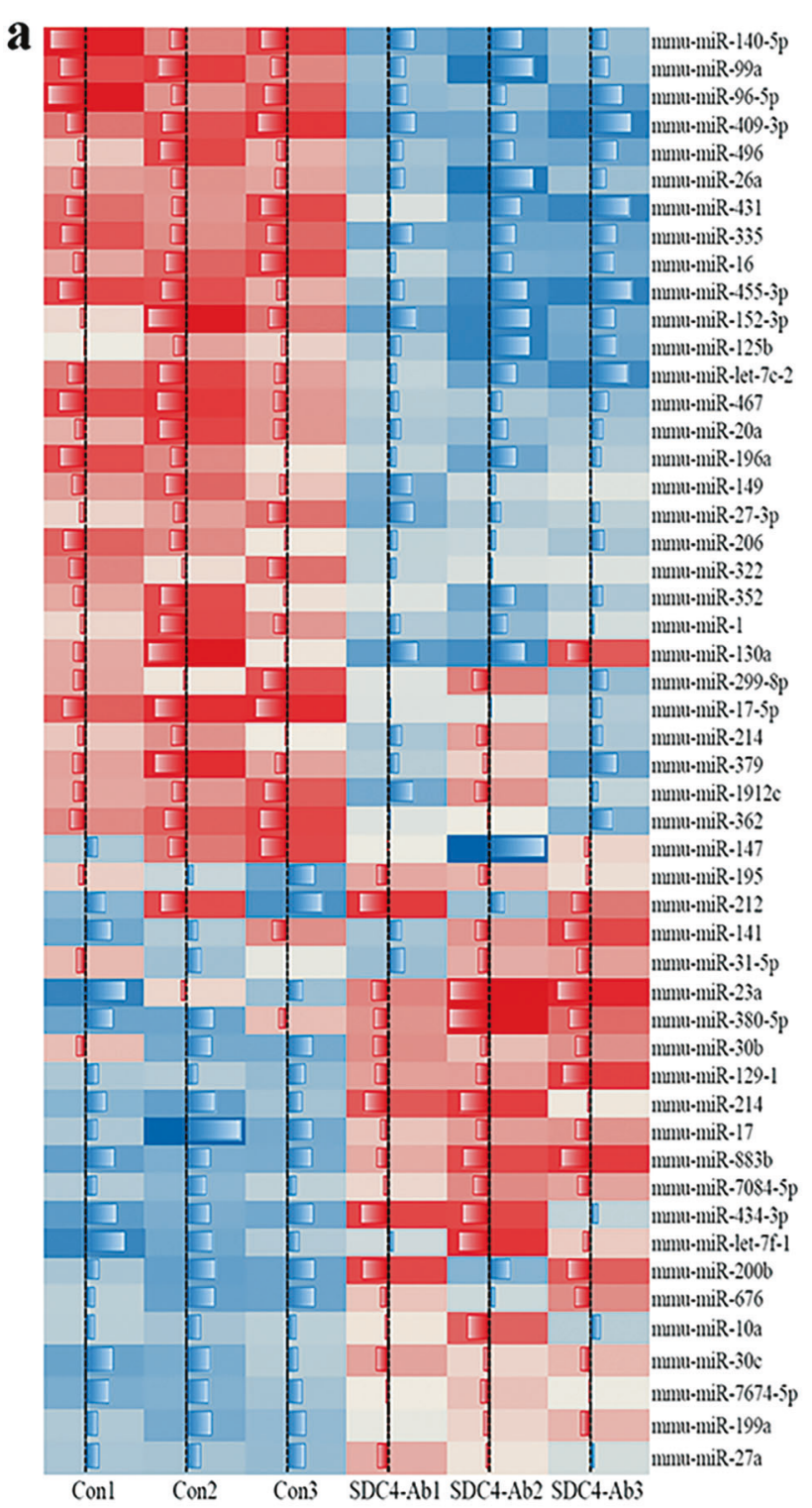

Fig. 2 The identification of osteoarthritis-related miRNAs in cartilage tissue and chondrocytes. a MiRNA microarray analysis of osteoarthritis-related candidate miRNAs. The heat map revealed the miRNAs whose expression levels were altered $>2$ - or $<2$-fold in cartilage tissue treated with $10 \mu \mathrm{l}$ of syndecan-4-specific antibody relative to the expression levels in control cartilage tissues. b Twenty-two candidate miRNAs were upregulated more than twofold in SDC-4

4 significantly decreased cartilage lesions in osteoarthritis at 2 and 4 weeks (Fig. 1a). Safranin O histological staining of cartilage tissue showed that extensive cartilage degradation and fibrosis occurred in the vehicle-treated group, whereas treatment with anti-SDC-4 significantly delayed the occurrence of cartilage destruction (Fig. 1b). In this study, using $\mathrm{X}$-ray, we also found that anti-SDC-4 significantly delayed cartilage destruction and degradation at 2 and 4 weeks (Fig. 1c). These results suggest that anti-SDC-4 can attenuate cartilage degradation and destruction in vivo.
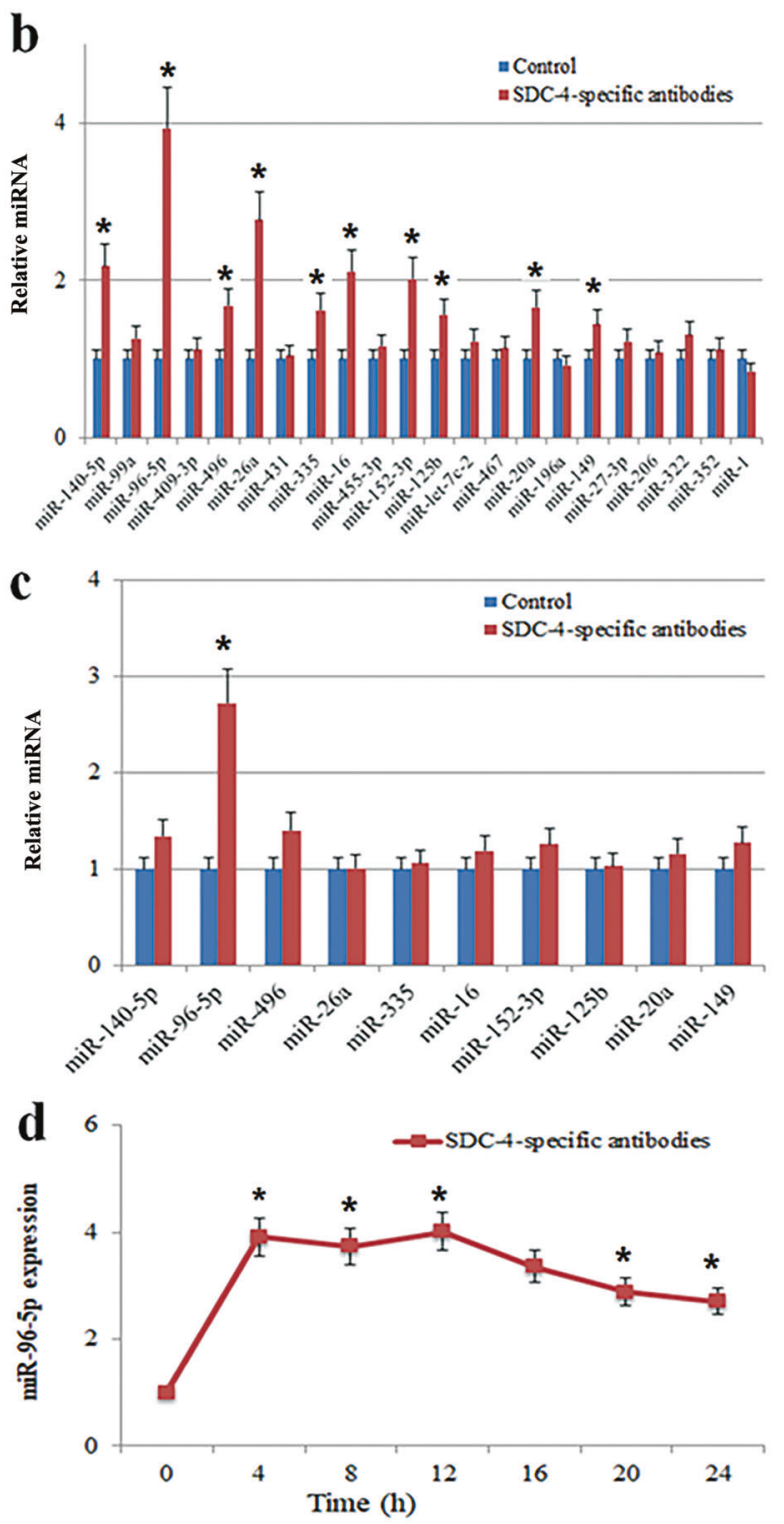

antibody-treated cartilage tissue. c MiR-140-5p, miR-96-5p, miR-496, miR-26a, miR-335, miR-16, miR-152-3p, miR-125b, miR-20a, and miR-149 were determined by RT-PCR in SDC-4 antibody-pretreated chondrocytes $(2 \mathrm{~h})$. d Time-course expression of the relative expression of miR-96-5p, as determined by real-time PCR, in chondrocytes exposed to SDC-4 antibodies $(24 \mathrm{~h})$. The values are expressed as the mean \pm SD from three independent experiments. $* P<0.05$.

Because dysregulation of chondrocyte function plays an important role in the pathogenesis of cartilage degradation and destruction $[3,4,6]$, we investigated the effects of anti-SDC-4 on the hypertrophic differentiation of chondrocytes in vitro. Information regarding the culture and identification of articular chondrocytes can be found in Supplementary Fig. 1. Articular chondrocytes obtained from the mouse knee joint were cultured with or without IL-1 $\beta$ in the absence or presence of SDC-4-specific antibodies for $48 \mathrm{~h}$ following $24 \mathrm{~h}$ of hypoxia. Using real-time 

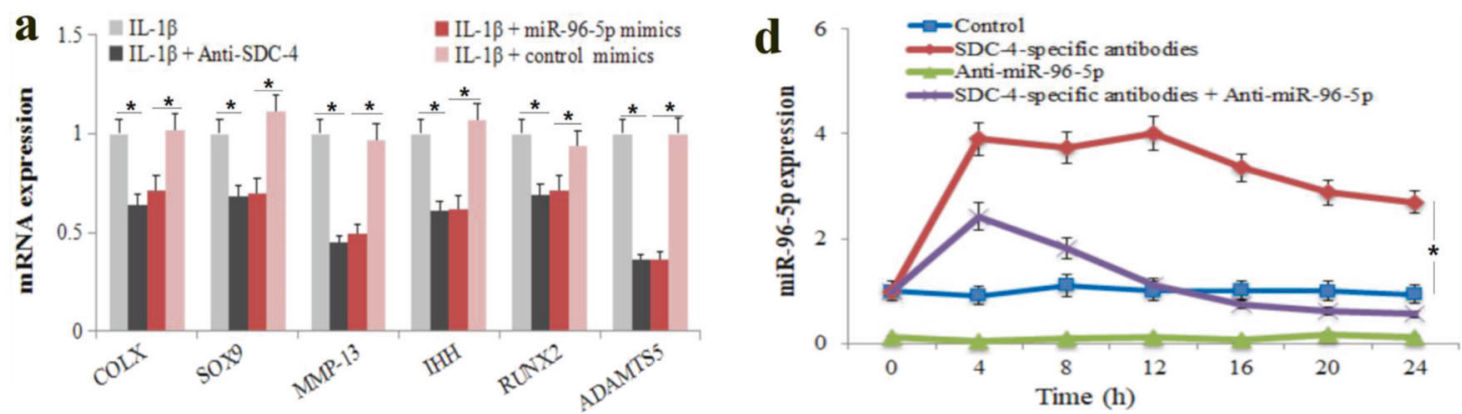

b

OA

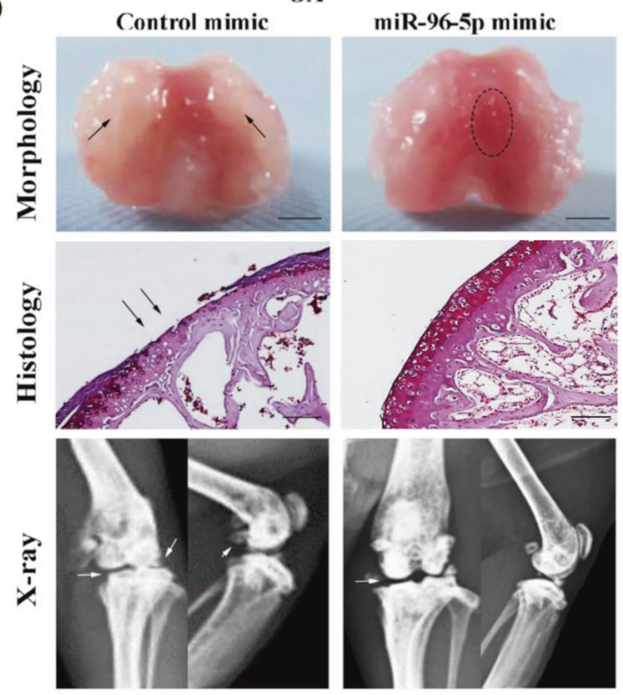

e

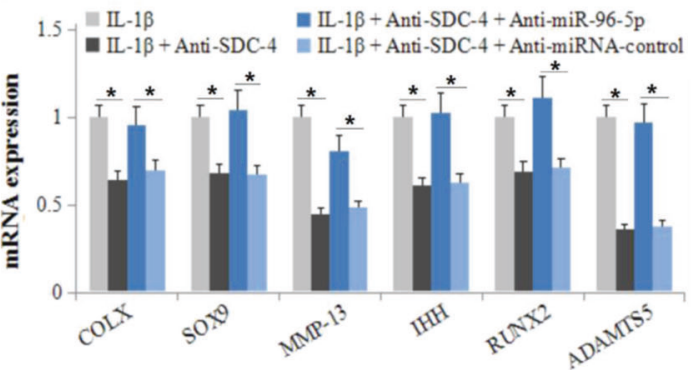

f

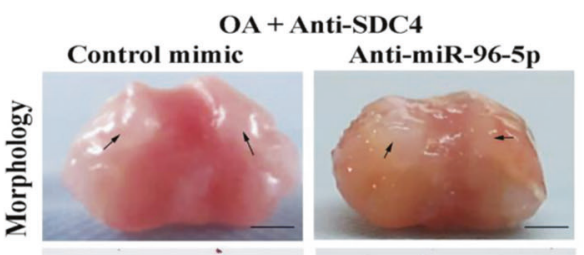

c
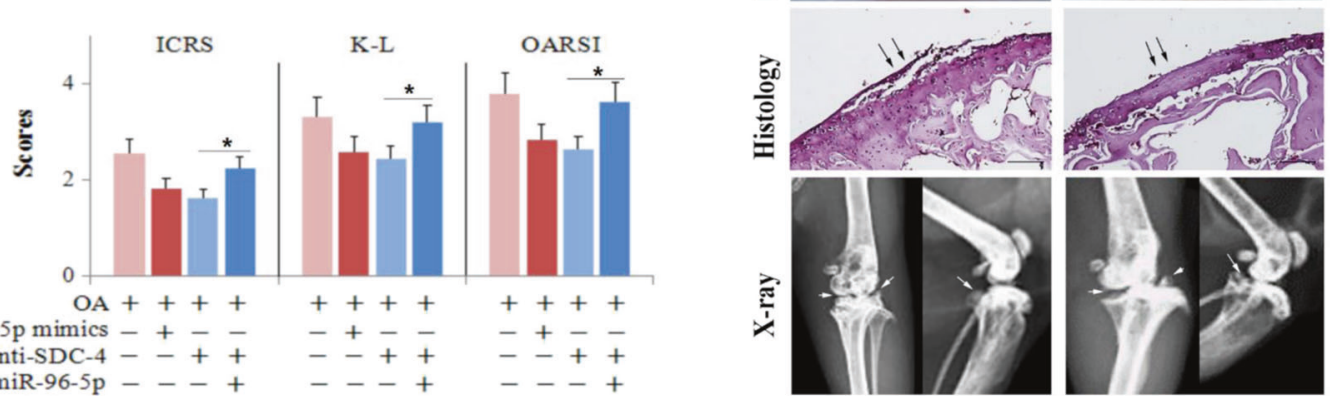

Fig. 3 MiR-96-5p mediates the effects of SDC-4-specific antibodies on murine osteoarthritis and chondrocyte hypertrophy. a Primary mouse chondrocytes were transfected with miR-96-5p mimics or control mimics for $24 \mathrm{~h}$, pretreated with the syndecan-4-specific antibody for $2 \mathrm{~h}$, and then stimulated with or without recombinant human IL-1 $\beta$ for $4 \mathrm{~h}$. The expression of COL-X, SOX9, MMP-13, IHH, RUNX2, and ADAMTS5 was determined by RT-PCR analyses in chondrocytes. b OA mice were administered $10 \mu 1$ miR-96-5p mimics or miRNA scrambled controls in the knee joint cavity. The dotted circles indicate wear area. The black arrows indicate chondrofibrosis. Bar $=2 \mathrm{~mm}$. Morphological, histological, and radiographic analyses of the femoral condyles were performed using a digital camera, safranin $\mathrm{O}$ staining, and X-ray at 4 weeks post operation. The black arrows indicate cartilage lesions. The white arrows indicate the degree of joint space narrowing and osteophytes. Bar $=$ $100 \mu \mathrm{m}$. c The cartilage lesions were graded on a scale of ICRS; the joint lesions were graded using the OARSI scoring system; and the radiographic changes were graded using the K-L scales. d, e Primary mouse chondrocytes were transfected with anti-miR-96-5p or anti-miRNA control for $24 \mathrm{~h}$, pretreated with the syndecan-4-specific antibody for $2 \mathrm{~h}$, and then stimulated with or without recombinant human IL- $1 \beta$ for $4 \mathrm{~h}$. The expression of miR-96-5p (d) and COL-X, SOX9, MMP-13, IHH, RUNX2, and ADAMTS5 (e) was determined by RT-PCR analyses in chondrocytes. f OA mice were administered $10 \mu \mathrm{l}$ of anti-miR-96-5p or anti-miRNA control in the knee joint cavity. Morphological, histological, and radiographic analyses of the femoral condyles were performed using a digital camera, safranin $\mathrm{O}$ staining, and $\mathrm{X}$-ray at 4 weeks post operation, respectively. The black arrows indicate chondrofibrosis and cartilage lesions. The white arrows indicate the degree of joint space narrowing and osteophytes. Bar $=2 \mathrm{~mm}$ (morphological) and $100 \mu \mathrm{m}$ (histological). The values are expressed as the mean \pm SD from three independent experiments. $* P<0.05$. 
$\mathbf{a}$

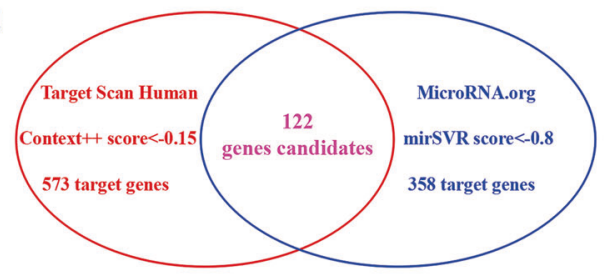

b miR-96-5p mimics $\quad$ Anti-miR-96-5p

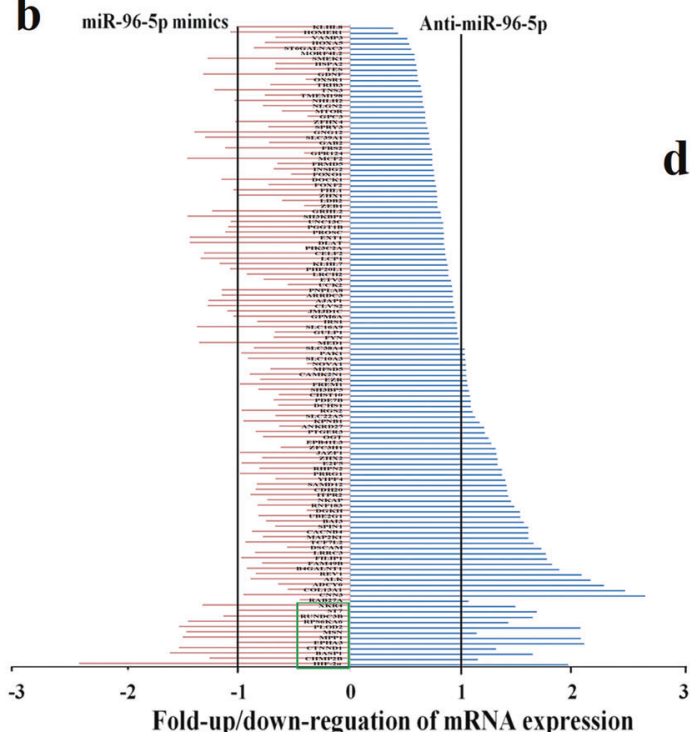

$\mathbf{f}$

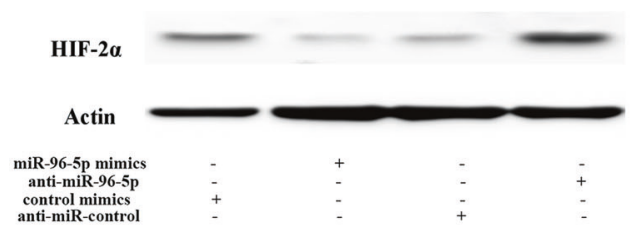

g

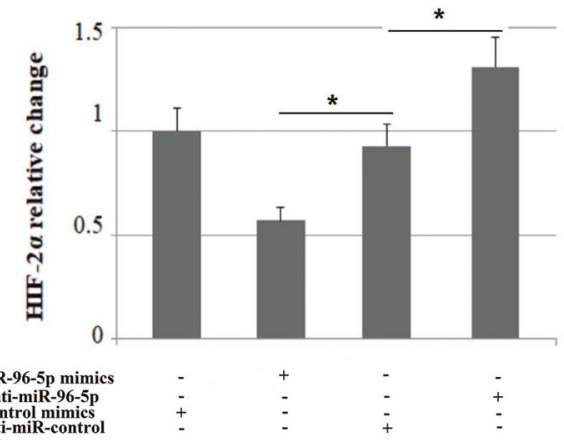

Fig. 4 The prediction and validation of miR-96-5p targets. a Online programs predict putative osteoarthritis-related target genes of miR96-5p. TargetScanHuman identified 573 target genes (Context ++ score $<-0.15)$, and MicroRNA.org identified 358 target genes (mirSVR score $<-0.8$ ). One hundred and twenty-two candidate's genes were designated putative target genes of miR-96-5p by both programs. b The expression of the 122 putative target genes was determined by real-time PCR in AMs transfected with miR-96-5p mimics or anti-miR-96-5p. c The expression of 12 potential target genes (HIF-2 $\alpha$, CHMP2B, BASP1, CTNND1 EPHA3, MPP1, MSN, PLOD2, RPS6KA6, RUNDC3B, ST7, and XKR4) was determined by real-time PCR in murine chondrocytes transfected with miR-96-5p

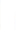
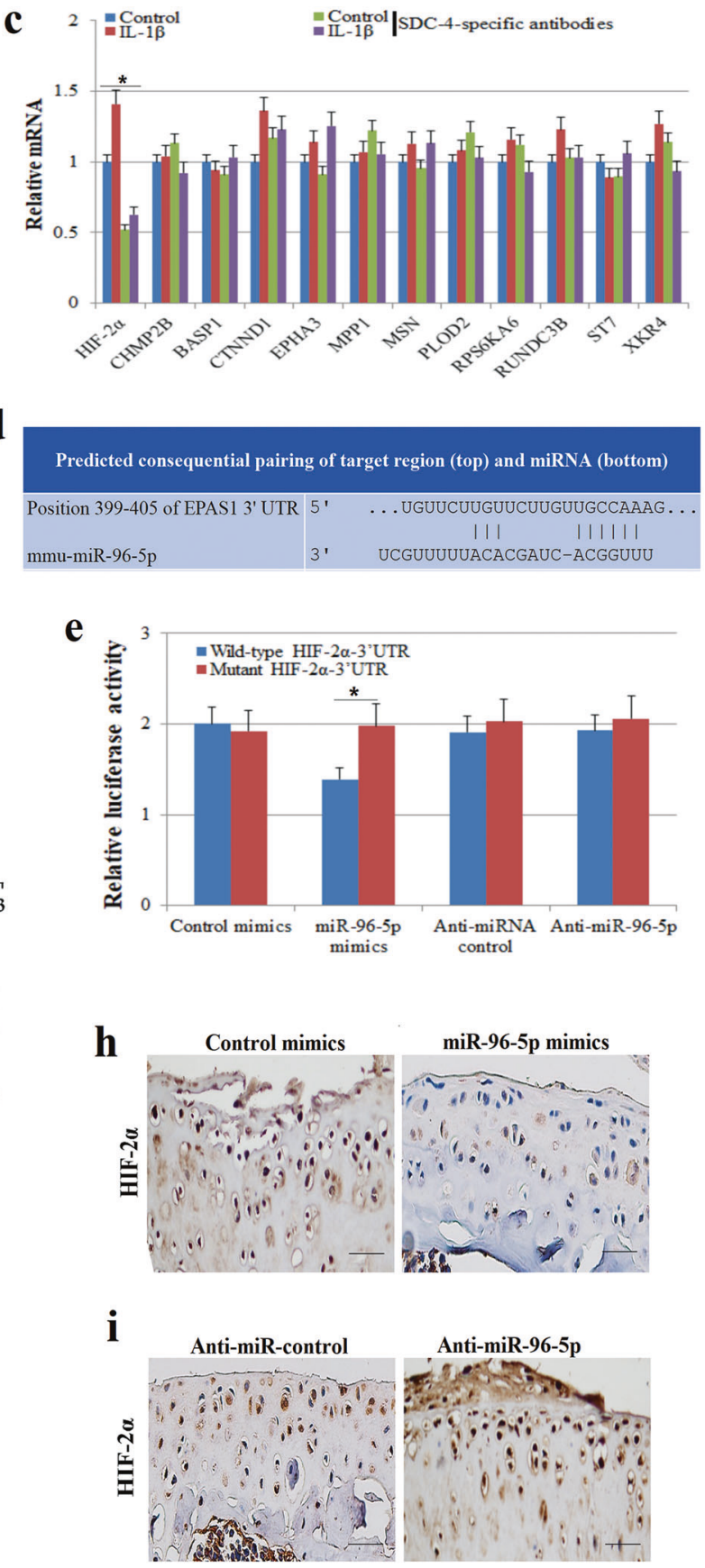

mimics or anti-miR-96-5p. d Predicted miR-96-5p binding sites in the 3 '-UTR of HIF-2 $\alpha$. e Luciferase reporter assay with cotransfection of wild-type or mutant HIF- $2 \alpha$ and miR-96-5p mimics, anti-miR-96-5p, mimics-control, or anti-miRNA-control in murine chondrocytes. $\mathbf{f}, \mathbf{g}$ Western blot analysis of HIF- $2 \alpha$ expression in chondrocytes transfected with miR-96-5p mimics, anti-miR-96-5p, mimic-control, or anti-miRNA-control. h, i Immunohistochemistry of HIF-2 $\alpha$ expression in cartilage tissues transfected with miR-96-5p mimics, anti-miR-96$5 \mathrm{p}$, mimic-control, or anti-miRNA-control. $\mathrm{Bar}=50 \mu \mathrm{m}$. All the treatments in this figure were carried out in triplicate, and the results are displayed as the mean $\pm \mathrm{SD}$. $* P<0.05$. 
PCR, we also found that anti-SDC-4 significantly inhibited the expression of transcription factors related to the processes of hypertrophy and phenotypic markers of cartilaginous hypertrophy, inducing the COL-X (Fig. 1d), SOX9 (Fig. 1e), MMP-13 (Fig. 1f), IHH (Fig. 1g), RUNX2 (Fig. 1h), and ADAMTS5 genes (Fig. 1i). The results showed that the inhibitory effect of SDC-4-specific antibodies on IL-1 $\beta$-induced chondrocytes was consistent with the in vivo results.

\section{The regulation of miRNA-96-5p expression by anti- SDC-4 treatment in cartilage tissue and chondrocytes}

Recent studies have shown that miRNAs play a pivotal role in OA development in vivo and in vitro by negatively regulating chondrocyte hypertrophy, thereby reducing cartilage degradation $[32,33]$. To identify potential miRNAs related to chondrocyte hypertrophy and cartilage degeneration, we performed a miRNA microarray analysis in antiSDC-4 cartilage tissue of osteoarthritic mice. Figure $2 \mathrm{a}$ shows that among the 51 miRNAs with expression levels that were altered by more than 1.5 -fold, 22 candidate miRNAs were upregulated in cartilage tissues from antiSDC-4 mice compared to the expression in control tissues. Real-time PCR confirmed that miR-140-5p, miR-96-5p, miR-496, miR-26a, miR-335, miR-16, miR-152-3p, miR125b, miR-20a, and miR-149 were upregulated in cartilage tissues from anti-SDC-4 antibody-treated mice $(P<0.05)$, and miR-96-5p was the most highly upregulated miRNA, which was consistent with the array results (Fig. 2b). We determined whether the treatment of chondrocytes with SDC-4-specific antibodies $(24 \mathrm{~h})$ could alter the expression of miR-96-5p compared with that of untreated chondrocytes. Consistent with the in vivo results, we also found that miR-96-5p was one of the ten miRNAs enriched in chondrocytes (Fig. 2c). The dynamic expression of miR-96$5 \mathrm{p}$ was examined after anti-SDC-4 treatment in chondrocytes. miR-96-5p expression increased by 3.91-fold after $4 \mathrm{~h}$ of treatment with SDC-4-specific antibodies and gradually decreased by $16 \mathrm{~h}$ in chondrocytes (Fig. 1d). These results suggested that miR-96-5p expression might be activated by anti-SDC-4 treatment in chondrocytes.

\section{Upregulation of miR-96-5p is essential for anti-SDC- 4-attenuated cartilage degradation and chondrocyte hypertrophy}

Anti-SDC-4 increased miR-96-5p expression in chondrocytes; therefore, we hypothesized that elevated levels of miR-96-5p may play an important role in cartilage degeneration and chondrocyte hypertrophy. We transfected chondrocytes and osteoarthritic cartilage tissue with a miR96-5p mimic or a control mimic and examined the chondrocyte phenotype after inducing IL-1 $\beta$ and cartilage degradation and destruction, which is associated with murine osteoarthritis. Similar to the anti-SDC-4 treatment, the miR-96-5p mimic significantly suppressed chondrocyte hypertrophy, downregulated the expression of hypertrophic chondrocyte markers (COL-X, SOX9, MMP-13, IHH, RUNX2, and ADAMTS5) (Fig. 3a), and suppressed cartilage degradation (Fig. 3b, c) at 4 weeks. Next, anti-miR-96$5 \mathrm{p}$ was transfected into chondrocytes and osteoarthritic cartilage tissue to inhibit the function of miR-96-5p. AntimiR-96-5p decreased both the basal and SDC-4-specific antibody-induced expression of miR-96-5p in chondrocytes (Fig. 3d). Moreover, anti-miR-96-5p inhibited the effects of anti-SDC-4 on chondrocyte hypertrophy (Fig. 3e) and cartilage degradation (Fig. 3f, c). These results indicate that anti-SDC-4-induced miR-96-5p is essential for inhibiting chondrocyte hypertrophy and attenuating cartilage degradation. This result raises the question of whether miR-96-5p might signal through multiple functionally and physically distinct targets.

\section{Predicting and validating the gene targets of $\mathrm{miR}$ - 96-5p that influence the effects of anti-SDC-4 in chondrocytes}

To examine the function of miR-96-5p in chondrocyte hypertrophy and cartilage degradation in greater detail, we used internet-based programs (Target Scan Human 7.1 and MicroRNA.org) to search for potential targets of miR96-5p and to identify the putative targets that might be related to OA development. We found 112 target candidates of miR-96-5p by both programs (Fig. 4a and Supplementary Table 1). The expression of 12 potential targets (HIF-2 $\alpha$, CHMP2B, BASP1, CTNND1 EPHA3, MPP1, MSN, PLOD2, RPS6KA6, RUNDC3B, ST7, and XKR4) was significantly affected by miR-96-5p expression modifications in murine chondrocytes by transfection with miR-96-5p mimics or anti-miR-96-5p (Fig. 4b). We found that only HIF- $2 \alpha$ expression was significantly inhibited by anti-SDC- 4 treatment in IL- $1 \beta$-induced chondrocytes (Fig. 4c), indicating that HIF-2 $\alpha$ might be related to OA development. We further confirmed that miR-96-5p can directly target the $3^{\prime}$-UTR of HIF- $2 \alpha$ by a luciferase assay (Fig. 4d, e). The expression of HIF-2 $\alpha$ was downregulated by miR-96-5p overexpression and upregulated by miR-96-5p silencing in chondrocytes (Fig. 4f, g) and osteoarthritis cartilage tissue (Fig. 4h, i), indicating that HIF- $2 \alpha$ is a direct target of miR-96-5p in murine chondrocytes and might be related to chondrocyte hypertrophy and cartilage degradation. 

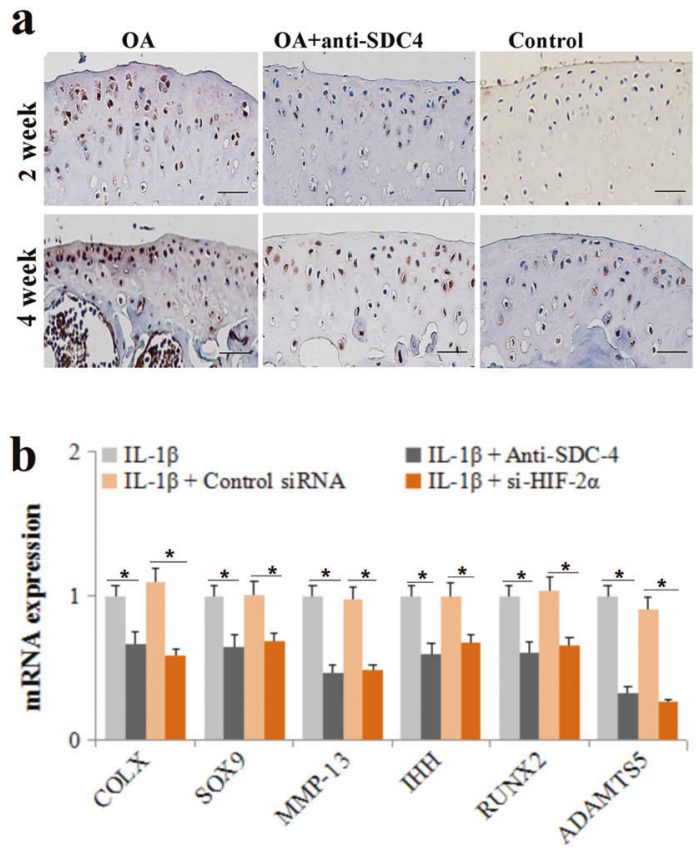

c

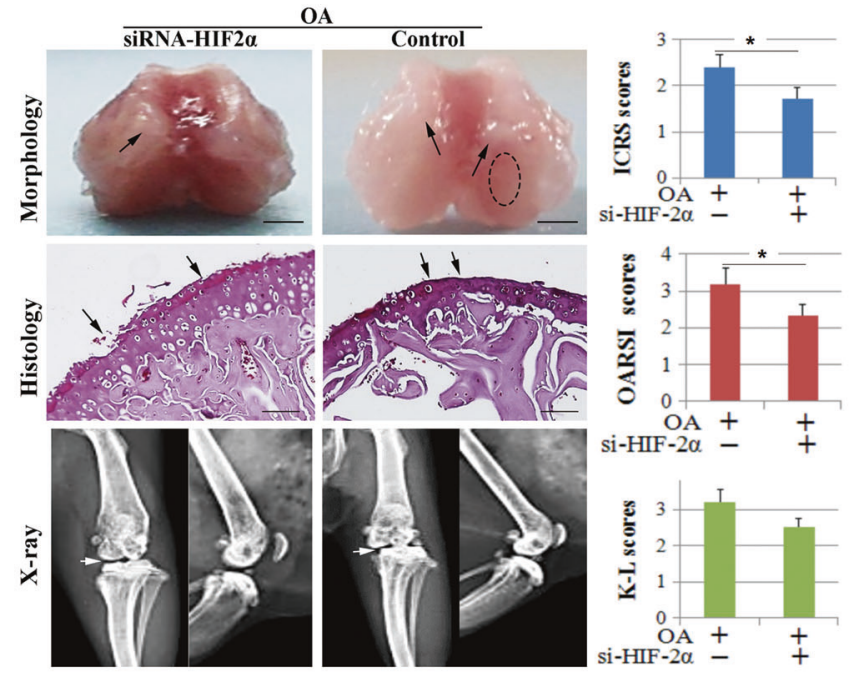

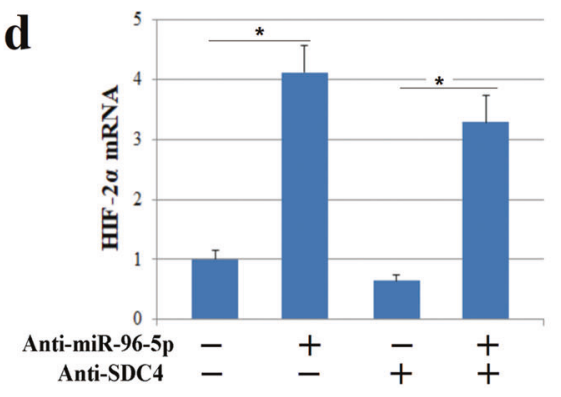

Fig. 5 HIF-2 $\alpha$ regulates the effects of SDC-4-specific antibodies and miR-96-5p on murine osteoarthritis and chondrocyte hypertrophy. a OA mice were injected with $10 \mu \mathrm{l}$ of SDC-4-specific antibody or an equivalent volume of saline into the knee joint cavity. Immunohistochemistry of HIF- $2 \alpha$ expression was performed in cartilage tissue at 2 and 4 weeks post operation. b Chondrocytes were transfected with si-HIF- $2 \alpha$ or control siRNA for $24 \mathrm{~h}$, pretreated with SDC-4-specific antibody for $2 \mathrm{~h}$ and then stimulated with or without recombinant human IL- $1 \beta$ for $4 \mathrm{~h}$. The expression of COL-X, SOX9, MMP-13, IHH, RUNX2, and ADAMTS5 was determined by RT-PCR analyses in chondrocytes. c OA mice were administered si-HIF- $2 \alpha$ or control siRNA in the knee joint cavity. Morphological, histological, and radiographic analyses of the femoral condyles were performed

\section{Anti-SDC-4-mediated inhibition of HIF-2a by miR-96 reduces chondrocyte hypertrophy and cartilage degradation}

HIF- $2 \alpha$ is a nuclear transcription factor that regulates the degradation of the cartilage matrix in osteoarthritic cartilage and mediates the hypertrophic differentiation of chondrocytes by regulating SOX9, IHH, RUNX2, and ADAMTS5 expression [22, 34]. We found that HIF-2 $\alpha$ expression was inhibited by SDC-4-specific antibodies in the cartilage tissue of a murine osteoarthritis model at 2 and 4 weeks by immunohistochemical staining (Fig. 5a). MiR- using digital camera, safranin $\mathrm{O}$ staining, and X-ray at 4 weeks post operation, respectively. The cartilage lesions were graded on a scale of ICRS; the joint lesions were graded using the OARSI scoring system; and the radiographic changes were graded using the $\mathrm{K}-\mathrm{L}$ scales. The dotted circles indicate wear area. The black arrows indicate chondrofibrosis and cartilage lesions. The white arrows indicate the degree of joint space narrowing. Bar $=2 \mathrm{~mm}$ (morphological) and $100 \mu \mathrm{m}$ (histological). d RT-PCR analysis of HIF- $\alpha$ expression in chondrocytes transfected with miR-96-5p mimics, anti-miR-96-5p, mimiccontrol or anti-miRNA-control, or/and SDC-4-specific antibody. e Immunohistochemistry analysis of ADAMTS5 expression in the cartilage tissue of murine osteoarthritis treated with miR-96-5p mimics, anti-miR-96-5p, or/and SDC-4-specific antibody. Bar $=50 \mu \mathrm{m}$.

96-5p can directly target the $3^{\prime}$-UTR of HIF- $2 \alpha$ and has been shown to inhibit the expression of HIF- $2 \alpha$ in murine chondrocytes. Anti-SDC-4 can induce miR-96-5p expression in chondrocytes, in addition to reducing cartilage matrix degeneration and IL-1 $\beta$-induced chondrocyte hypertrophy. Therefore, we hypothesized that the anti-SDC4-dependent suppression of cartilage degradation and chondrocyte hypertrophy might be mediated through downregulation of HIF-2 $\alpha$ by miR-96-5p. We decreased the endogenous level of HIF- $2 \alpha$ by siRNA (si-HIF- $2 \alpha$ ) in murine chondrocytes and osteoarthritis cartilage tissue and then measured the hypertrophic differentiation of 
chondrocytes and cartilage degradation. Transfection with si-HIF-2 $\alpha$ significantly decreased the expression of COL-X, SOX9, MMP-13, IHH, RUNX2, and ADAMTS5 in chondrocytes (Fig. 5b) and suppressed cartilage degradation and destruction in murine osteoarthritis (Fig. 5c). These results suggest that the downregulation of HIF- $2 \alpha$ inhibited chondrocyte hypertrophy and cartilage degradation to a similar extent as the anti-SDC- 4 or miR-96-5p treatments. HIF- $2 \alpha$ expression was inhibited by SDC-4-specific antibodies or miR-96-5p in chondrocytes (Fig. 5d). ADAMTS5, a downstream factor that is regulated by HIF- $2 \alpha$, showed the same inhibition by anti-SDC-4 or miR-96-5p treatment as HIF-2 $\alpha$ (Fig. 5e). When endogenous miR-96-5p was depleted by anti-miR-96-5p, anti-SDC-4 treatment neither repressed the expression of HIF-2 $\alpha$ (Fig. 5f) nor inhibited chondrocyte hypertrophy and cartilage degeneration (Fig. 3d, e). Thus, the anti-SDC-4-dependent improvement of cartilage degeneration and chondrocyte hypertrophy appears to be mediated by the downregulation of HIF- $2 \alpha$ by miR-96-5p.

\section{Discussion}

Several studies have confirmed that SDC-4 is strongly expressed in OA chondrocytes, and increasing levels of SDC-4 expression are correlated with the severity of osteoarthritis-like histopathological injuries [11-13]. A study conducted by Echtermeyer et al. [13] and Barre et al. [10] showed that knockout of the SDC-4 gene or injection of SDC-4-specific inhibitory antibodies into the articular cavity can reduce the expression of MMP-3 and ADAMTS5, alleviate cartilage matrix degradation, and protect articular cartilage in OA rats. Our data were similar to previous reports that showed that SDC-4 expression increased gradually in a mouse OA model. At week 4 post operation, knee joint cartilage damage was significantly alleviated in the anti-SDC-4 group compared to that in the OA group. These results indicated that SDC-4 expression is related to $\mathrm{OA}$ progression and that inhibiting SDC-4 expression could delay OA development.

In contrast to HIF- $1 \alpha$, HIF- $2 \alpha$ is a catabolic factor in the osteoarthritic process $[22,35]$. HIF- $2 \alpha$ is mainly expressed in highly differentiating chondrocytes as a key transcription factor that mediates degradation of the cartilage matrix and the hypertrophic differentiation of chondrocytes in human and murine osteoarthritic cartilage [21, 36]. Several stimulating factors, such as stress stimulation and the inflammatory response, act on chondrocytes to influence HIF- $2 \alpha$ expression and to further mediate the pathological process of OA by regulating SOX9, IHH, and RUNX2 expression, thereby stimulating the expression of the matrix-degrading enzyme ADAMTS5 [34]. In our study, HIF-2 $\alpha$ expression gradually increased within 4 weeks post operation and then declined with the progression of osteoarthritis. This confirmed that HIF- $2 \alpha$ plays an important role in OA, and the trend is consistent with the trend that was previously reported in a study conducted by Yang et al. [22] and Satio et al. [4], which showed that HIF- $2 \alpha$ increases joint instability in the aged knees of rats in the OA model during the early stage of the disease. According to our results, the increase in HIF-2 $\alpha$ expression can be suppressed during the early stage of OA after inhibiting the function of SDC-4. These results validate our hypothesis that SDC-4 mediates the expression of HIF- $2 \alpha$.

Recently, several miRNAs have been shown to influence cartilage homeostasis, and their relationship with OA development has also received increasing attention [24, 25, 28, 29]. For example, miR-26a, miR-16, miR-132, miR-146a, and miR-223 expression levels are decreased in osteoarthritis [24, 37]. MiR-27a, miR-27b, and miR-146a appear to attenuate ADAMTS and/or MMP expression in cartilage [38, 39]. The identification of key miRNA networks in osteoarthritic cartilage could provide long-awaited diagnostic, therapeutic, and prognostic tools for early detection, improved treatment, and extended life spans and may support the best possible quality of life for patients with osteoarthritis. Therefore, we identified potential miRNAs related to cartilage degeneration and chondrocyte hypertrophy using miRNA microarray analysis, which showed that miR-96-5p expression was upregulated in chondrocytes and cartilage tissue treated with SDC-4specific antibodies. The importance of microRNAs in cartilage homeostasis is also supported by a study showing that Sox 9 positively regulates COL2A1 expression in chondrocytes by a miR-96-5p-dependent mechanism [37]. More importantly, we found that the upregulation of miR-96-5p is essential for anti-SDC-4-attenuated cartilage degeneration and chondrocyte hypertrophy.

An improved understanding of the mechanisms of diseases could facilitate early detection and effective treatments. We used internet-based programs to search for the potential targets (112 putative target genes) of miR-96-5p. We found that 12 potential targets (including HIF- $2 \alpha$ ) were significantly affected by the corresponding miR-96-5p expression modifications. However, HIF- $2 \alpha$ expression was significantly inhibited by anti-SDC-4 treatment in IL$1 \beta$-induced chondrocytes by examining the 12 potential targets, indicating that HIF- $2 \alpha$ might be related to OA development. We further confirmed that miR-96-5p can directly target the $3^{\prime}$-UTR of HIF- $2 \alpha$ to inhibit the expression of HIF-2 $\alpha$ in murine chondrocytes. Moreover, we confirmed that the anti-SDC-4-dependent alleviation of cartilage degradation and chondrocyte hypertrophy appears to be mediated by the downregulation of HIF- $2 \alpha$ by miR96-5p. 
This study had a few limitations. First, although it differs from the processes by which OA develops clinically, we used the classic surgically induced OA model in this study. Many factors can be attributed to OA, such as trauma, inflammation, and aging. Therefore, the surgically induced OA model cannot fully simulate the pathogenesis of OA. Second, we focused mainly on the effects of anti-SDC-4 and miR-96-5p on chondrocytes and cartilage in the study and did not identify their effects on other cells or tissues in the knee joint. We are planning to investigate the role of anti-SDC-4 and miR-96-5p in the synovium and subchondral bone of the OA model, and the related signal transduction pathways will be identified. Third, the study period was 4 weeks and could only simulate the clinical course of early to middle-stage OA. In future studies, we will investigate the expression and regulation of SDC-4 and HIF- $2 \alpha$ in late-stage OA.

In summary, we demonstrated that anti-SDC- 4 antibody treatment slows the progression of cartilage degradation and inhibits IL-1 $\beta$-induced chondrocyte hypertrophy in a murine osteoarthritis model. MiR-96-5p expression in chondrocytes and cartilage tissue was upregulated by anti-SDC-4- and anti-SDC-4-attenuated IL-1 $\beta$-induced chondrocyte hypertrophy and the progression of cartilage destruction by a miR-96-5p-dependent mechanism in murine osteoarthritis. MiR-96-5p can directly target the $3^{\prime}$-UTR of HIF- $2 \alpha$ to inhibit HIF-2 $\alpha$ signaling in murine chondrocytes. Overall, our study demonstrated that the inhibition of SDC-4 reduces cartilage degradation in murine models of osteoarthritis through the downregulation of HIF- $2 \alpha$ by miR-96-5p and provides a potential therapeutic strategy for osteoarthritis.

\section{Data availability}

All data generated or analyzed during this study are included in this published article (and its Supplementary information files).

Author contributions $\mathrm{KZ}$ and $\mathrm{ZZ}$ performed study concept and design; $\mathrm{KZ}, \mathrm{ZZ}$, and FP performed development of methodology and writing, review, and revision of the paper; $\mathrm{KZ}, \mathrm{HY}$, and $\mathrm{SH}$ provided acquisition, analysis, and interpretation of data and statistical analysis; $\mathrm{SH}$ provided technical and material support. All authors read and approved the final paper.

Funding The study was supported by the Sichuan Science and Technology Department Key Research Projects (No. 2019YFS0129), the China Postdoctoral Science Foundation (No. 2019M653419), the Post-Doctor Research Project, West China Hospital, Sichuan University (No. 2018HXBH049), and the National Natural Science Foundation of China (No. 82002304). The funding source had no role in the study design; the acquisition, analysis, or interpretation of the data; the writing of the paper; or the decision to submit the paper for publication.

\section{Compliance with ethical standards}

Conflict of interest The authors declare no competing interests.

Ethical approval This study did not require ethical approval.

Publisher's note Springer Nature remains neutral with regard to jurisdictional claims in published maps and institutional affiliations.

\section{References}

1. Felson DT. Clinical practice. Osteoarthritis of the knee. N Engl J Med. 2006;354:841-8.

2. Varela-Eirin M, Loureiro J, Fonseca E, Corrochano S, Caeiro JR, Collado M, et al. Cartilage regeneration and ageing: targeting cellular plasticity in osteoarthritis. Ageing Res Rev. 2018;42:56-71.

3. Goldring MB, Goldring SR. Articular cartilage and subchondral bone in the pathogenesis of osteoarthritis. Ann N Y Acad Sci. 2010;1192:230-7.

4. Saito T, Fukai A, Mabuchi A, Ikeda T, Yano F, Ohba S, et al. Transcriptional regulation of endochondral ossification by HIF2alpha during skeletal growth and osteoarthritis development. Nat Med. 2010;16:678-86.

5. van den Berg WB. Osteoarthritis year 2010 in review: pathomechanisms. Osteoarthr Cartil. 2011;19:338-41.

6. Luyten FP, Lories RJ, Verschueren P, de Vlam K, Westhovens R. Contemporary concepts of inflammation, damage and repair in rheumatic diseases. Best Pract Res Clin Rheumatol. 2006;20:829-48.

7. Heinegard D, Saxne T. The role of the cartilage matrix in osteoarthritis. Nat Rev Rheumatol. 2011;7:50-6.

8. Sachs N, Sonnenberg A. Cell-matrix adhesion of podocytes in physiology and disease. Nat Rev Nephrol. 2013;9:200-10.

9. Stewart MD, Sanderson RD. Heparan sulfate in the nucleus and its control of cellular functions. Matrix Biol. 2014;35:56-9.

10. Barre PE, Redini F, Boumediene K, Vielpeau C, Pujol JP. Semiquantitative reverse transcription-polymerase chain reaction analysis of syndecan- 1 and -4 messages in cartilage and cultured chondrocytes from osteoarthritic joints. Osteoarthr Cartil. 2000;8:34-43.

11. Binch ALA, Shapiro IM, Risbud MV. Syndecan-4 in intervertebral disc and cartilage: saint or synner? Matrix Biol. 2016;52-54:355-62.

12. Pap T, Bertrand J. Syndecans in cartilage breakdown and synovial inflammation. Nat Rev Rheumatol. 2013;9:43-55.

13. Echtermeyer F, Bertrand J, Dreier R, Meinecke I, Neugebauer K, Fuerst M, et al. Syndecan-4 regulates ADAMTS-5 activation and cartilage breakdown in osteoarthritis. Nat Med. 2009;15:1072-6.

14. Matsui Y, Iwasaki N, Kon S, Takahashi D, Morimoto J, Matsui Y, et al. Accelerated development of aging-associated and instabilityinduced osteoarthritis in osteopontin-deficient mice. Arthritis Rheum. 2009;60:2362-71.

15. Kon S, Ikesue M, Kimura C, Aoki M, Nakayama Y, Saito Y, et al. Syndecan-4 protects against osteopontin-mediated acute hepatic injury by masking functional domains of osteopontin. J Exp Med. 2008;205:25-33.

16. Fujita N, Hirose Y, Tran CM, Chiba K, Miyamoto T, Toyama Y, et al. HIF-1-PHD2 axis controls expression of syndecan 4 in nucleus pulposus cells. Faseb J. 2014;28:2455-65.

17. Ummarino D. Osteoarthritis: hypoxia protects against cartilage loss by regulating Wnt signalling. Nat Rev Rheumatol. 2016;12:315. 
18. Lin N, Simon MC. Hypoxia-inducible factors: key regulators of myeloid cells during inflammation. $\mathrm{J}$ Clin Investig. 2016;126:3661-71.

19. Taylor CT, Doherty G, Fallon PG, Cummins EP. Hypoxiadependent regulation of inflammatory pathways in immune cells. J Clin Investig. 2016;126:3716-24.

20. Colgan SP, Campbell EL, Kominsky DJ. Hypoxia and mucosal inflammation. Annu Rev Pathol. 2016;11:77-100.

21. Higgs R. Osteoarthritis: HIF-2alpha drives osteoarthritis development. Nat Rev Rheumatol. 2010;6:435.

22. Yang S, Kim J, Ryu JH, Oh H, Chun CH, Kim BJ, et al. Hypoxiainducible factor-2alpha is a catabolic regulator of osteoarthritic cartilage destruction. Nat Med. 2010;16:687-93.

23. Yang S, Ryu JH, Oh H, Jeon J, Kwak JS, Kim JH, et al. NAMPT (visfatin), a direct target of hypoxia-inducible factor- $2 \alpha$, is an essential catabolic regulator of osteoarthritis. Ann Rheum Dis. 2015;74:595-602.

24. Etich J, Holzer T, Pitzler L, Bluhm B, Brachvogel B. MiR-26a modulates extracellular matrix homeostasis in cartilage. Matrix Biol. 2015;43:27-34.

25. Seidl CI, Martinez-Sanchez A, Murphy CL. Derepression of microRNA-138 contributes to loss of the human articular chondrocyte phenotype. Arthritis Rheumatol. 2016;68:398-409.

26. Vicente R, Noël D, Pers YM, Apparailly F, Jorgensen C. Deregulation and therapeutic potential of microRNAs in arthritic diseases. Nat Rev Rheumatol. 2016;12:211-20.

27. Meng F, Li Z, Zhang Z, Yang Z, Kang Y, Zhao X, et al. MicroRNA-193b-3p regulates chondrogenesis and chondrocyte metabolism by targeting HDAC3. Theranostics. 2018;8:2862-83.

28. Miyaki S, Asahara H. Macro view of microRNA function in osteoarthritis. Nat Rev Rheumatol. 2012;8:543-52.

29. Kung LHW, Ravi V, Rowley L, Angelucci C, Fosang AJ, Bell $\mathrm{KM}$, et al. Cartilage microRNA dysregulation during the onset and progression of mouse osteoarthritis is independent of aggrecanolysis and overlaps with candidates from end-stage human disease. Arthritis Rheumatol. 2018;70:383-95.

30. Gosset M, Berenbaum F, Thirion S, Jacques C. Primary culture and phenotyping of murine chondrocytes. Nat Protoc. 2008;3:1253-60.

31. Thysen S, Luyten FP, Lories RJ. Targets, models and challenges in osteoarthritis research. Dis Model Mech. 2015;8:17-30.

32. Miyaki S, Sato T, Inoue A, Otsuki S, Ito Y, Yokoyama S, et al. MicroRNA-140 plays dual roles in both cartilage development and homeostasis. Genes Dev. 2010;24:1173-85.

33. Dudek KA, Lafont JE, Martinez-Sanchez A, Murphy CL. Type II collagen expression is regulated by tissue-specific miR-675 in human articular chondrocytes. J Biol Chem. 2010;285:24381-7.

34. Zhang FJ, Luo W, Lei GH. Role of HIF-1alpha and HIF-2alpha in osteoarthritis. Joint Bone Spine. 2015;82:144-7.

35. Bouaziz W, Sigaux J, Modrowski D, Devignes CS, Funck-Brentano $\mathrm{T}$, Richette $\mathrm{P}$, et al. Interaction of HIF1 $\alpha$ and $\beta$-catenin inhibits matrix metalloproteinase 13 expression and prevents cartilage damage in mice. Proc Natl Acad Sci USA. 2016;113:5453-8.

36. Ryu JH, Shin Y, Huh YH, Yang S, Chun CH, Chun JS. Hypoxiainducible factor- $2 \alpha$ regulates Fas-mediated chondrocyte apoptosis during osteoarthritic cartilage destruction. Cell Death Differ. 2012;19:440-50.

37. Murata K, Yoshitomi H, Tanida S, Ishikawa M, Nishitani K, Ito $\mathrm{H}$, et al. Plasma and synovial fluid microRNAs as potential biomarkers of rheumatoid arthritis and osteoarthritis. Arthritis Res Ther. 2010;12:R86.

38. Li X, Gibson G, Kim JS, Kroin J, Xu S, van Wijnen AJ, et al. MicroRNA-146a is linked to pain-related pathophysiology of osteoarthritis. Gene. 2011;480:34-41.

39. Akhtar N, Rasheed Z, Ramamurthy S, Anbazhagan AN, Voss FR, Haqqi TM. MicroRNA-27b regulates the expression of matrix metalloproteinase 13 in human osteoarthritis chondrocytes. Arthritis Rheumatol. 2010;62:1361-71. 